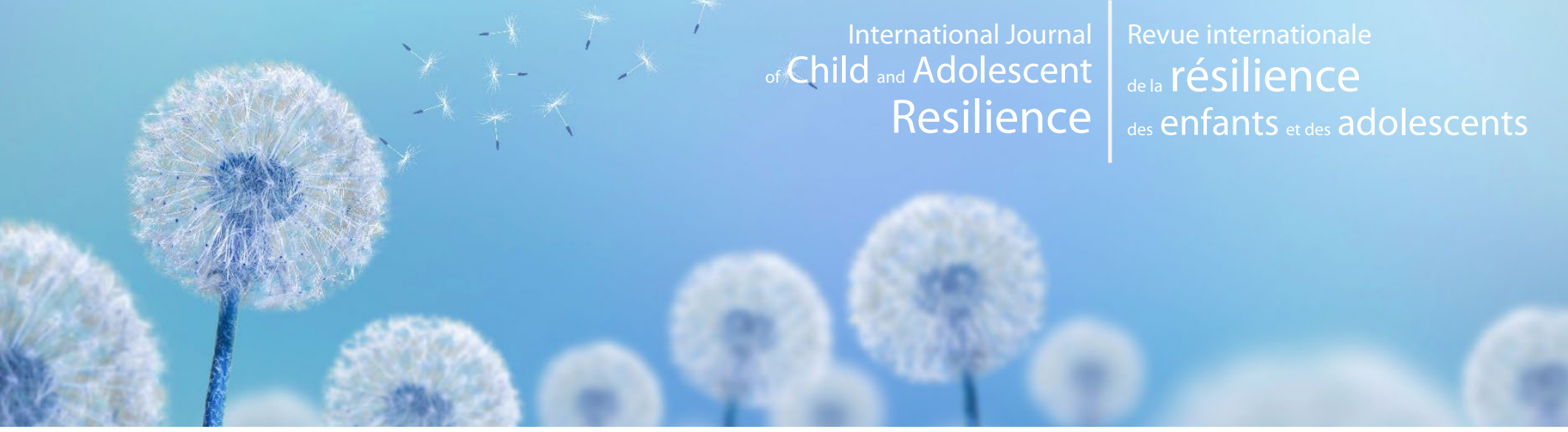

\title{
Trauma-informed care implementation in the child- and youth-serving sectors: A scoping review
}

\section{Ash LOWENTHAL ${ }^{1}$}

1 School of Social Work, McGill University

Corresponding Author: Ash Lowenthal, School of Social Work, McGill University, 3506 University St., Montréal, Québec, Canada H3A 2 A7.

Email: ashley.lowenthal@mail.mcgill.ca.

\section{Abstract}

Objectives: Enthusiasm for trauma-informed care (TIC) in the child-and youth-serving sectors (CYSSS) has been growing dramatically over the last decade. However, TIC implementation activity on the ground has far outpaced research and the landscape of TIC implementation scholarship is not well known. This scoping review aims to explore: 1) the nature of TIC implementation research in the CYSSs; 2) the characteristics of the change initiatives being studied; 3) the types of evidence these studies have generated; and 4) the gaps in the literature.

Methods: On August 28, 2019, the EBSCO, Scopus, Web of Science and PsycINFO databases were searched for Englishlanguage, peer-reviewed articles that mentioned "trauma-informed" and ("child" or "children" or "adolescent" or "youth") in the title, abstract or keywords. Articles selected for this review reported on TIC implementation processes in the CYSSs. Fifty-four articles published between 2004 and 2019 met the inclusion criteria and were reviewed in-depth for this scoping review.

Results: High variability was found in the characteristics of TIC implementation research and practice. However, promising preliminary evidence is beginning to show that TIC implementation can lead to a reduction in violent practices and incidents and can improve service provider knowledge, attitude, behaviour and practice (KABP). However, research shows that improvements to self-reports of KABP are often modest, not always maintained over time and not easily translated into actual changes in behaviour, practice and organizational climate due to a variety of barriers.

Conclusion: In order to address these barriers, the preliminary evidence suggests that TIC implementation requires a comprehensive approach that includes commitment from senior leadership, ongoing support, and collaboration within and between service providing organizations and systems.

Keywords: Trauma-informed care; children and youth; implementation; organizational change. 


\section{Introduction}

Violence against children and youth has many different forms. Whether it is in the form of abuse, neglect or exposure to structural violence, it can leave a lasting impact on children and youth's health and well-being. The traumainformed care (TIC) movement has been mobilizing knowledge about the pervasive and potentially lifelong impacts these forms of adversity can have on children and youth since the early 2000s. Since then, complex trauma has emerged as a framework for understanding these types of interpersonal, chronic, and cumulative adversities, as well as the associated constellation of sequalae that can derail all domains of child development (Cloitre et al., 2009; Courtois, 2008; van der Kolk et al., 2005). In addition to promoting a greater understanding of children and youth who have faced adversity and may be experiencing complex trauma, the TIC movement has been advocating for service providers to better equip themselves to understand and respond to their needs. As a result, there is a growing recognition that conventional service systems often fail to respond appropriately to the needs of complex trauma-impacted children and youth and may also cause further harm (Bloom \& Farragher, 2010, 2013; Elliot et al., 2005; Harris \& Fallot, 2001; Oudshoorn, 2015). In order to meaningfully implement the principles of TIC in the various sectors that serve children, youth and their families, there is a general consensus that a major shift in organizational culture, structure, and policy is required.

Unlike trauma-specific interventions (TSIs), which include various treatment modalities such as TraumaFocused Cognitive Behavioral Therapy (TF-CBT), TIC is much broader in its scope. It is a service delivery paradigm that aims to inform every level of operations. TIC implementation is a systems-level intervention that aims to transform the environment in which services are provided. The implementation of TSIs can be a part of a broader initiative to adopt TIC within an agency or service system. However, TSI implementation alone is not considered TIC implementation if it is not also accompanied by systems-level interventions that aim to shift the culture, structure or policy of the provider of the TSI.

While the concept of TIC is rather contested and amorphous, TIC is often defined using the parameters set out by the Substance Abuse and Mental Health Services Administration (2014), which state that a trauma-informed organization:

realizes the widespread impact of trauma and understands potential paths for recovery; recognizes the signs and symptoms of trauma in clients, families, staff, and others involved with the system; and responds by fully integrating knowledge about trauma into policies, procedures, and practices, and seeks to actively resist re-traumatization (p. 9).

However, the TIC framework has many different interpretations and is continuously evolving. For example, researchers have developed the trauma- and violence-informed care (TVIC) framework to strengthen the understanding and response to structural violence, which refers to the harm caused by colonialism, racism, heteronormativity, poverty, ableism, etc., which is lacking in many TIC approaches (Levine et al., 2020). The TVIC framework will also support a more robust interrogation into the ways that service providers are complicit in the perpetuation of structural violence.

The core principles of TIC in child- and youth-serving sectors (CYSSs) promote a shift away from a focus on compliance and pathology towards a focus on connection, empathy and deeper understanding (Bloom \& Farragher, 2010, 2013; Ford \& Blaustein, 2013; Oudshoorn, 2015). For example, in residential or inpatient contexts, supporting a young person to de-escalate aggressive behaviour through empathic listening and emotion regulation practices is now considered to be safer and more therapeutic than using punitive, coercive and pharmacological approaches such as time-outs and physical and/or chemical restraints. The science is clear that strong relationships with adult caregivers are a key factor of resilience in the face of adversity and complex trauma, and the adoption of TIC is crucial for service providers to be able to foster these types of relationships with the children and youth in their care (Blaustein \& Kinniburgh, 2010; Bryson et al., 2017; Ford \& Blaustein, 2013). In light of this, TIC is increasingly becoming associated with best practice in a variety of service contexts, especially in CYSSs where early intervention is considered key to preventing the complex and long-term sequelae associated with early adversity. Various levels of governments in Canada, the United States, and Australia have enacted policy that commits to trauma-informed service delivery across a variety of sectors in the human service system.

However, with all these commitments to provide trauma-informed services, some have commented on the resulting emergence of a booming TIC consultation and training industry. As Birnbaum (2019) states "it would not be an exaggeration to suggest that TIC training has become a mostly unregulated growth industry, as there is little 
systematic research on the content or quality of these proliferating offerings" (p. 477). Many have commented that all the work happening on the ground to operationalize TIC has far outpaced research (Hanson \& Lang, 2016; Thomas et al., 2019). Indeed, the study of TIC implementation is an area of scholarship that is new but growing rapidly and the landscape of the associated research literature is not well known.

\section{Objectives}

As of yet, there exists no other comprehensive reviews of TIC implementation research across the CYSSS. A 2020 systematic review from Purtle looked at TIC implementation across all sectors of the human service system and focused on quantitative, outcome-based research. Two systematic reviews with a focus on the youth residential treatment sector exist: one with a focus on outcomes (Bailey et al., 2019), the other with a focus on process (Bryson et al., 2017). Furthermore, all existing reviews found a high level of heterogeneity in the literature, pointing to the complexity of grasping the nature of research being conducted in this field and the need for a scoping review to map out this scholarship. This scoping review bridges the silos in the CYSSs as well as in research by examining TIC implementation research of all types across all CYSSs. Importantly, it also maintains the boundary with the adult-serving sector to retain the important developmental considerations to trauma-informed service delivery with young people. The objective of this scoping review is to provide a comprehensive overview of the research about TIC implementation in the CYSSs by: 1) describing the key characteristics of TIC implementation research studies; 2) describing the key characteristics of TIC implementation initiatives in the CYSSs being studied; 3) mapping the types of evidence available about implementation outcomes and processes; and 4) identifying important gaps in the literature.

\section{Method}

A scoping review is indicated for mapping evidence in a body of literature that is heterogenous and has yet to be comprehensively reviewed (Arksey \& O'Malley, 2005; Levac et al., 2010; Munn et al., 2018; Peters et al., 2015). Scoping reviews are designed to explore the extent, range, and nature of research activity, summarize key findings, and identify gaps. Methodological guidelines for scoping reviews recommend iterative study designs to allow for the refinement of inclusion and exclusion criteria as the researcher becomes more familiar with the literature and develops a better sense of the volume, breadth and nature of the scholarship (Arksey \& O'Malley, 2005; Levac et al., 2010; Peters et al., 2015). This study followed the iterative six-stage scoping review framework developed by Arksey and O'Malley (2005): 1) identifying the research question; 2) identifying relevant studies; 3) selecting studies; 4) charting the data; 5) collating summarizing and reporting the results; and 6 ) engaging in consultation. While the study was limited by only having one reviewer complete stages 1 through 5, it was strengthened by consulting with a committee of other TIC researchers throughout the process to validate the methods and findings.

During stage 1, the following research question was identified: What is the nature of the TIC implementation research literature in the CYSSs? In addition, parameters of what TIC implementation research in the CYSSs consists of were developed, with the expectation they would be refined as the selection process progressed. Initiating stage 2, a search was conducted on August 28, 2019 of the Academic Search Complete (EBSCO), Scopus, Web of Science and PsycINFO (OVID) databases for English-language journal articles that mentioned "trauma-informed" and "child" or "adolescent" or "youth" in the title, abstract or key words. The search was not filtered for publication date. A total of 2306 records were obtained from this database search and imported into Endnote. Following the removal of duplicates, 1152 unique articles were then screened by title, abstract and full-text review in an iterative process that ultimately generated the final inclusion/exclusion criteria.

After becoming more familiar with the results of the search, the original parameters of TIC implementation research in the CYSSS were narrowed to include only the studies that reported on the results of a TIC implementation initiative. Studies that were simply evaluating to what degree organizations and systems were already operating in a trauma-informed way and studies that took baseline measures of TIC uptake or readiness for implementation were excluded. Studies focused on evaluating only the implementation of a TSI were also excluded. In regard to parameters of the CYSSs, articles were excluded if they did not explicitly mention the inclusion of child- and youth-serving professionals, organizations, or systems in their sample. Articles selected for this literature review studied at least one aspect of the change process associated with TIC implementation at all scales, including those that targeted only one department or unit of an institution, and those that aimed to make changes to entire organizations or service systems. Studies were included regardless of quality, as is indicated for scoping reviews, although some conclusions were drawn 
about how to improve the quality of research in this area moving forward. Fifty-four articles met the inclusion criteria and were reviewed in-depth for this paper.

In stage 4, data were systematically abstracted and charted into an excel sheet using Garrard's Matrix Method (2017) with the following headings: author, title, year of publication, journal title, sector (e.g. Child welfare, youth residential treatment, schools, etc.), geographic location (country and region) where research took place, aims of the study, methodology, measures/data collection method, data analysis method, sample, implementation model(s) utilized, TIC model(s)/framework(s)/guide(s) utilized, types of systems-level interventions used (e.g. training, policy change, leadership development etc.), key results, and implications for research and practice. However, as discussed later on in the paper, articles were not always forthcoming with all these details about their research and it was not always possible to extract data for all these categories of analysis. In stage 5, data was collated and summarized numerically and thematically in order to provide a comprehensive overview of all the material reviewed in a way that best responded to the research question and objectives.

\section{Results}

\section{Characteristics of the research studies}

\section{Overarching methodological approach}

Table 1 classifies the types of articles that were included in this review by overarching methodological design. Quantitative research clearly dominated the scholarship. Moreover, the overwhelming majority of quantitative studies represented outcome-based, prepost test research designs. The mixed methods studies were also largely outcome-based, pre-post test designs that complemented this type of data with focus groups and/or interviews with staff. Descriptive, commentary

Table 1. Articles Reviewed by Research Design

\begin{tabular}{llc}
\hline \multirow{2}{*}{ Research design } & \multicolumn{2}{c}{ Articles reviewed } \\
\cline { 2 - 3 } & $\boldsymbol{n}$ & $\boldsymbol{\%}$ \\
\hline Descriptive & 5 & 9 \\
Qualitative & 5 & 9 \\
Mixed Methods & 12 & 22 \\
Quantitative & 32 & 60 \\
\hline
\end{tabular}

54

100 and theoretical articles that either advocated for the adoption of TIC, provided a theoretical framework for TIC or described TIC implementation initiatives made up a large part of the TIC literature reviewed during the screening process. Five quasi-empirical, descriptive articles were included because they blurred the distinctions between empirical and descriptive by providing valuable information about various aspects of TIC implementation processes from the perspective of researchers who participated in them (Akin et al., 2017; Collin-Vézina et al., 2019; Fraser et al., 2015; McCorkle et al., 2005; Regan, 2010). These papers often provided insights into the implementation process that the rest of the outcome-focused literature had difficulty capturing.

The over-representation of quantitative, outcome-focused studies may reflect a willingness to prove that TIC "works" and generate evidence to back up the movement's calls for its widespread adoption. It may also reflect the hegemony of evidence-based practice's (EBP) hierarchy of evidence, which seemed to pervade this scholarship. When studies discussed how and why they chose their methodology and recommendations for future research, they often referenced how experimental research, especially the randomized control trial (RCT), was the gold-standard for rigorous evidence and that more RCTs were needed to validate the effectiveness of TIC. Only one study in the review actually used the RCT method, (Hoysted et al., 2019) and perhaps not coincidentally, it evaluated the shortest, most limited implementation initiative of all the studies: a 15-minute online TIC training program for pediatric emergency department staff. Several other studies used other experimental or quasi-experimental designs, but tensions and contradictions emerged between the pressure to produce what EBP considers gold-standard evidence and the suitability of experimental methods for studying TIC implementation. In discussing their rationale for not choosing an experimental method, Murphy et al. (2017) state that "it became clear that training all staff in TST [trauma systems therapy] and facilitating their fidelity to the TST model is not an event; rather it is an iterative process that takes place over time and requires substantial investment, coordination and effort" (p. 25). TIC implementation researchers may need to grapple with whether RCTs or other types of experimental research are appropriate for studying such complex, emergent and iterative phenomena. If there continues to be pressure to conduct experimental, outcome-based research, research that could generate more nuanced, rich data about implementation processes will continue to be de-prioritized. It may also be important to consider forms of research that better address the complex sociopolitical context of TIC implementation and/or meaningfully address equity issues and power dynamics at play. 


\section{Publication date}

Data regarding the publication dates of articles selected revealed important information about the history of publication trends in this scholarship. "Using Trauma Theory to design service systems", the seminal theoretical anthology that first coined the term "trauma-informed care", was published in 2001 by Harris and Fallot. After this, empirical literature on TIC implementation slowly began to appear in the mid-2000s. Two other highly influential theoretical texts were published in the early 2010's: the Attachment, Regulation and Competency (ARC) framework manual in 2010 by Blaustein and Kinniburgh, and the Sanctuary Model manual in 2013 by Bloom and Farragher; these were followed by an exponential growth in the empirical literature on TIC in the CYSSs from 2014 to the present. Figure $1 \mathrm{graphs}$ the number of articles that were selected for this in-depth review by the year they were published. Likely due to the much higher volume of TIC studies that are being published as of late, the majority of the articles reviewed for this paper were published in the last three years.

Figure 1. Articles Reviewed by Year Published.

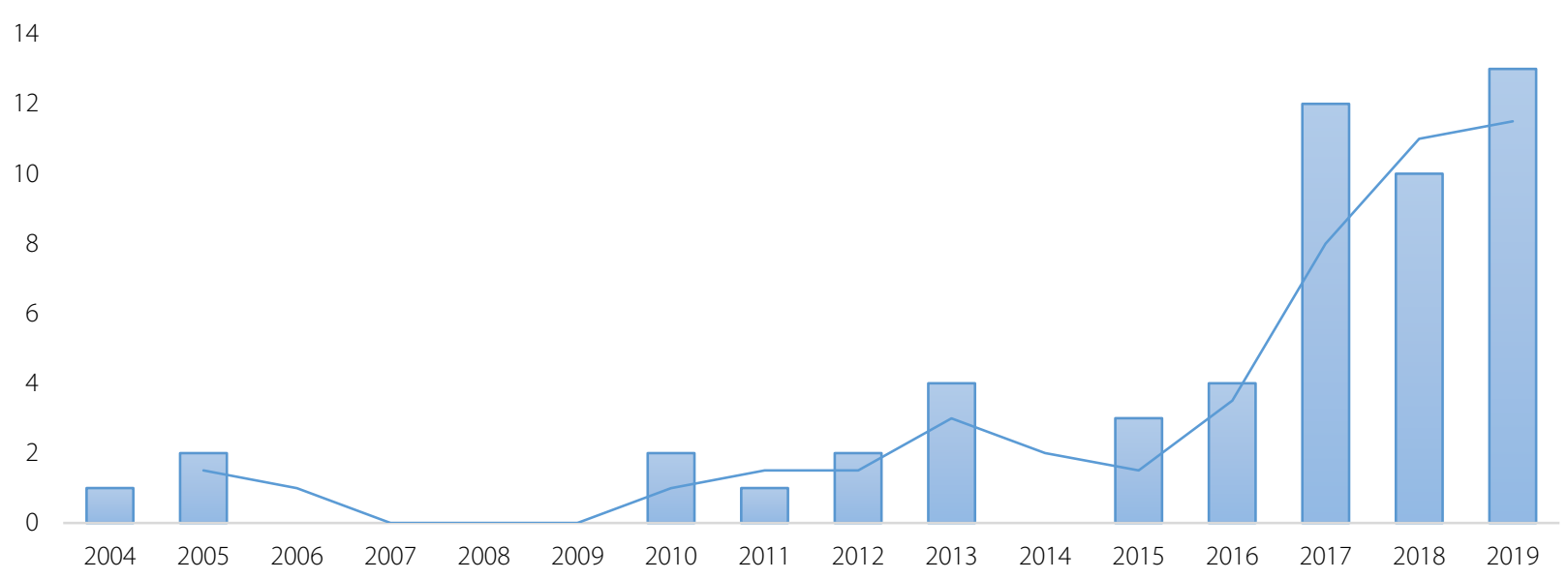

\section{Geographic location}

Table 2 illustrates how TIC scholarship is overwhelmingly concentrated in the United States, with 87\% of articles meeting inclusion criteria reporting on US studies. This should not come as a surprise given that the concept of TIC was developed in the United States, but it remains clear that there are gaps in knowledge around its applicability and relevance in other contexts. Studies categorized as "International" conducted research in multiple geographic locations that crossed the state borders of Canada, Australia and the United States as well as Scotland and New Zealand.

Table 2. Articles Reviewed by Location of Study

\begin{tabular}{lcc}
\hline \multirow{2}{*}{ Country } & \multicolumn{2}{c}{ Articles Reviewed } \\
\cline { 2 - 3 } & $\boldsymbol{n}$ & $\%$ \\
\hline Canada & 2 & 4 \\
Australia & 2 & 4 \\
International & 3 & 5 \\
United States & 47 & 87 \\
\hline Total & $\mathbf{5 4}$ & $\mathbf{1 0 0}$ \\
\hline
\end{tabular}

Table 3. Articles Reviewed by Sector

\begin{tabular}{lcc}
\hline \multirow{2}{*}{ Sector } & \multicolumn{2}{c}{ Articles Reviewed } \\
\cline { 2 - 3 } & $\boldsymbol{n}$ & \% \\
\hline School & 3 & 6 \\
Community Mental Health & 4 & 7.5 \\
Inpatient Psychiatric & 4 & 7.5 \\
Health & 5 & 9 \\
Other & 5 & 9 \\
Multisectoral & 5 & 9 \\
Residential Treatment & 13 & 24 \\
Child Welfare & 15 & 28 \\
\hline Total & $\mathbf{5 4}$ & $\mathbf{1 0 0}$ \\
\hline
\end{tabular}




\section{Service sector}

Table 3 lays out the various CYSSs represented in the articles selected for an in-depth review. Studies from the child welfare and adolescent residential treatment sectors made up over half of the literature reviewed for this paper and seem to be the sectors with the most robust TIC implementation evidence base. The studies categorized as "Other" had less than three published studies meeting inclusion criteria within their sector. These studies represented the diversity of sectors engaging in TIC implementation and included family drug treatment court, gang intervention, home visiting, and youth justice. This hints at the widespread interest in TIC but also suggests there may be limited penetration of TIC within these diverse service sectors to date. Studies categorized as "Multisectoral" collected data from professionals and organizations across different sectors.

There were many multisectoral TIC studies in the search results that were screened and excluded because they did not specify explicitly whether they included child- and youth-serving professionals or organizations. This may reflect how there has yet to be a strong emphasis in the TIC literature about the important developmental considerations of trauma-informed service delivery with young people, as well as the unique methodological considerations for studying TIC with children and youth. Even in the literature that was selected and reviewed for this paper, TIC frameworks that were developed either for the adult-serving sector or ones that were developed to be universal were often applied to the CYSSs without adaptation. This fails to address the ways that trauma, especially complex trauma, can derail healthy development and ignores the need for TIC to address gaps in core developmental competencies in complex trauma-impacted children and youth (Blaustein \& Kinniburgh, 2010; Perry, 2009). Developmental science has found that development occurs sequentially, with each competency laying the foundation for increasingly complex competencies. A trauma-informed approach with children should include these developmental considerations. The ARC model (Blaustein \& Kinniburgh, 2010) seems to be among the few TIC frameworks that meaningfully integrates the findings of developmental science in their approach.

However, ARC targets the therapeutic relationship between staff and the children and youth in their care. While systemic in certain aspects, the framework is not comprehensive enough to provide guidance for creating and sustaining organizational culture, structure and policy that could support ARC implementation. The theoretical literature is clear that TSIs must be implemented within an organizational context that is trauma-informed in order for service users to safely access them. As ARC straddles the divide between a TSI and a systems-level intervention, initiatives implementing ARC have had to borrow from other frameworks with more of a focus on organizational development to support implementation initiatives. Yet, none of these organizational development frameworks are geared towards CYSSs, which may suggest that one does not yet exist. This remains an important gap in the TIC literature and demonstrates how TIC may not yet be fully adapted for the CYSSs in ways that sufficiently incorporate child development science as well as organizational development theory. It points to the lack of comprehensive TIC models that incorporate guidance on all aspects of operations of trauma-informed service provision with young people, from the more micro aspects like intervention approaches to the more macro aspects such as organizational policy, an issue that will be further explored throughout the paper.

\section{Characteristics of the implementation initiatives}

\section{Scale and scope of the implementation initiatives}

Consistent with the findings of other reviews, there was a high degree of variability in the scale and scope of TIC implementation initiatives studied by the articles included in this review, between and within sectors. However, child welfare tended to have the largest initiatives in scale and scope, while pediatric health care generally had the smallest. In order of the smallest scope to the largest scope, the initiatives ranged from:

1. one-off, staff training-only initiatives with durations ranging from 15 minutes to several days;

2. initiatives only implementing a new trauma screening protocol;

3. initiatives providing ongoing TIC training, coaching, supervision, and leadership development;

4. initiatives that combined TIC training with ongoing support, coaching, and/or supervision, and changes to organizational culture, structure, policy and physical environment.

Some initiatives were very small in scale, studying a small agency or a single department within a larger organizational context, while others encompassed entire citywide or statewide child-and youth-serving systems of care. A post hoc analytical frame, entitled the TIC Implementation Scope Continuum, was developed to classify the wide range of TIC implementation initiatives. It is informed by the theoretical TIC implementation literature that 
recommends taking a comprehensive, multi-stage, whole-system approach (Bloom \& Farragher, 2013; Elliott et al., 2005; Harris \& Fallot, 2001; Hopper et al., 2010).

As pictured in Figure 2, the left end of the continuum represents limited change initiatives ( $\mathrm{LCls}$ ) which make up about one third of the studies, where the TIC implementation activities (TIAs) were brief, narrow in scope and targeted only one or two levels of organizational operations. These generally consisted of one-off trainings with little to no follow-up and few, if any, interventions to address the physical environment and/or organizational culture, structure and/or policy. The right end represents comprehensive change initiatives (CCls) which make up about another third of the studies that used numerous different strategies over longer periods of time to create changes in the organizational culture, structure and policy as well as the broader service system in order to support whole-system $\mathrm{TIC}$. In the middle were the other initiatives that made moderate efforts to address organizational culture, structure and policy using a few different types of interventions over a moderate period of time. These were termed moderate change initiatives (MCls). There was a relatively even breakdown of studies classified as $\mathrm{LCls}, \mathrm{MCl}$ and CCls in the sample of articles reviewed, with about one third within each of the three categories along the TIC Implementation Scope Continuum.

Figure 2. TIC Implementation Scope Continuum

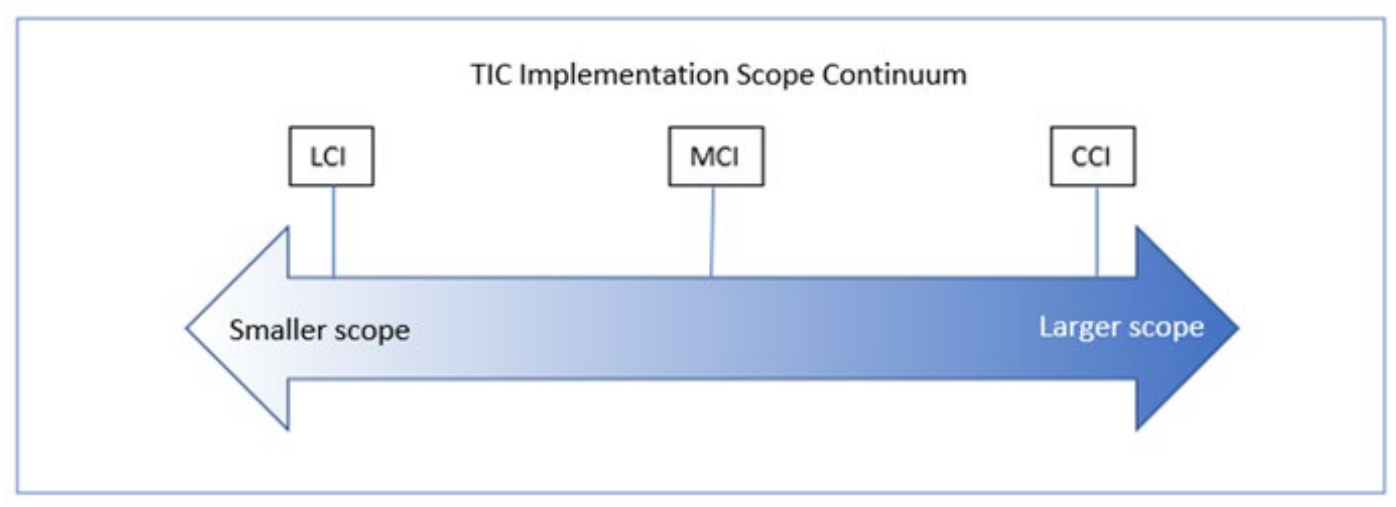

Limitations of LCls. The relatively even spread of initiatives across the continuum points to the high diversity of TIC implementation initiatives. It also suggests that most initiatives do not have the resources or the theoretical grounding to take a comprehensive, whole-system approach to implementation. There is a growing recognition that training alone, especially training that is short, one-off and didactic, has a limited impact (Baker et al., 2018; Beidas \& Kendall, 2010; Denison et al., 2018; Williams \& Smith, 2017). Also, Lang et al. (2016) state that "as interest in traumainformed care grows, there is a risk that 'receiving some trauma-related training' becomes equivalent to 'being traumainformed'. However, we believe that trauma training is an essential but not nearly sufficient element of a traumainformed system" (p. 122). There is growing evidence that training-only interventions have limited impacts on observable changes in practice, beyond those that are self-reported (Beidas \& Kendall, 2010; Damian et al., 2019; Williams \& Smith, 2017). In one study, Goetz and Taylor-Trujillo (2012) found that shifting practice to become more trauma-informed was challenging, with staff often "reverting to previous and familiar interventions" post-training and that "consistency, reinforcement, and support for the new interventions were a continuous process" (p. 102). Damian et al. (2019) found that while workers' knowledge of TIC increased after training, they felt that their capacity to provide TIC to their clients did not. These workers reported feeling disempowered to make change within their organizational culture, policy and structure due to a lack of buy-in from upper management. Meaningful change requires ongoing work and the participation of all levels of the organizational hierarchy to be sustained, especially in complex service systems where change is difficult and where the dominant service delivery paradigm may be incompatible with TIC principles (Bryson et al., 2017; Jankowski et al., 2018; McCorkle et al., 2005; Regan, 2010).

TIC implementation may need to be more clearly and emphatically defined as an organizational transformation process and an emphasis made on how training alone does not accomplish this. Moreover, it may be advisable for LCls like short, one-off TIC trainings, to not use the term "TIC implementation" at all to refer to their initiatives and studies. It may be more accurate for LCl studies to use terms likes "trauma training evaluation" or "trauma screening evaluation" and this may help address the issue of heterogeneity in the literature. As it could be argued that 
LCls do not encompass the crucial facets of TIC implementation as defined by the theoretical literature, the term "implementation" was not used to define the various scales ( $\mathrm{LCls}, \mathrm{MCl}$ and CCls) of the TIC implementation Scope Continuum. It may be advisable for this field to come to a consensus that TIC implementation is necessarily an endeavour that requires more than just training or just screening and that $\mathrm{LCls}$ using only these interventions should not be considered "TIC implementation".

Characteristics of $\mathrm{CCls}$. To contrast the articles studying $\mathrm{LCls}$, there were over a dozen articles in the review that studied large US TIC implementation projects, and several articles reporting on different aspects of the same massive change initiatives (Conners-Burrow et al., 2013; Damian et al., 2017, 2018; 2019; Murphy et al., 2017; Redd et al., 2017). For example, two articles (Barto et al., 2018; Fraser et al., 2015) studied the Massachusetts Child Trauma Project (MCTP), a large five-year statewide, multi-sectoral systems-improvement initiative. The Massachusetts's child welfare system collaborated with two mental health agencies and two large, urban medical centers to positively influence complex trauma-impacted young people's safety, permanency, and well-being; objectives that were common to most child welfare-based initiatives. In this sample of studies, multisectoral collaboration in the context of a $\mathrm{CCl}$ often took place between the child welfare and pediatric mental health-care systems.

The MCTP was guided by Trauma Informed Leadership Teams (TILTs), a leadership model that developed by the National Child Traumatic Stress Network's (NCTSN). TILTs, made up of major stakeholders from the child- and youthserving system of care, including families who access services, were tasked with "installing and supporting a structure for TIC systems integration at the community level" by enhancing coordination, collaboration and adopting a shared language for understanding complex trauma-impacted children and youth (Bartlett et al., 2016, p. 102). Several other TIC projects (Azeem et al., 2011; Collin-Vézina et al., 2019; Drabble et al., 2013; Esaki et al., 2018; Jankowski et al., 2018; Middleton et al., 2015) used similar models of leadership development and collaboration but, used a diverse set of terms to refer to these strategies. The development of a consensus on TIC terminology for leadership development strategies may be helpful. In the MCTP, the TILTs efforts to create organizational and systemic change were guided by SAMHSA's concept of trauma and guidance for a trauma-informed approach, a guide that was used among a large number of the initiatives in this review (Substance Abuse and Mental Health Services Administration, 2014).

Proportion of the workforce reached. Demonstrating the wide reach of the MCTP, Barto et al. (2018) report that the MCTP had trained $71 \%$ of the state's child welfare workers, with a quarter of those receiving advanced training, and had launched TILTs in 27 out of 29 regional child welfare agencies. Reporting on the proportion of the workforce reached by trainings or other implementation activities was not common, as articles would often state the number of staff members trained but not what percentage of the total workforce. This data would provide valuable information regarding the reach of TIA's in a department, organization or service system.

\section{TIC models, guides, frameworks and measures used}

The MCTP represents one of the most robust of organizational and systemic change initiatives in this sample of articles, with different types of interventions at various levels of the entire state's CYSSs. It also seemed to be one of the most theoretically grounded, referencing pre-established models of leadership, workforce development, training curriculum, TSIs and trauma-informed organizational culture, structure, and policy. While dozens of other studies also employed some of the same frameworks used by the MCTP, there were a dozen other TIC guides, models or frameworks cited and the combinations of models used in the initiatives was almost never identical to others. Moreover, few used a pre-established, and/or theoretically grounded approach for each of these aspects of implementation. Many initiatives created their own content for their trainings, their own workforce development strategy, their own TIC implementation strategies and their own measures to evaluate the initiative, while some failed to cite any guides, models or approaches they were informed by. There are likely barriers to accessing TIC models and measures, such as financial constraints, but open source TIC guides do exist. However, as mentioned previously, none of the existing guides seemed comprehensive enough to provide recommendations for each aspect, component and level of TIC implementation, from the micro, to the mezzo, to the macro. Because the guides all target different aspects of practice, training, and implementation as well as organizational policy, culture, structure and systemic change, they needed to be combined in order to provide guidance for each component of a TIC implementation process. It may be helpful for the field of TIC scholarship to consider developing resources for implementation in the CYSSs that are more comprehensive.

Transparency. Further complicating reviews of this literature, authors often only provided scant details about the initiatives being studied. They often omitted crucial information about if and how pre-existing TIC guides, models 
and frameworks informed implementation, the content and approach of the training(s) provided, the specifics about other implementation activities, and the reach within the organization. The lack of information and clarity about the initiatives being studied represents an important limitation of this literature, a critique shared by Purtle (2020) in their systematic review. Further TIC implementation research should strive to be as transparent as possible about each aspect of the change initiative they are studying.

\section{Types of evidence available}

\section{Satisfaction and self-reported knowledge, attitudes, behaviour and practice change}

Cross-sectional studies. Satisfaction with TIC implementation activities as well as self-reports of TIC-related knowledge, attitudes, behaviour and practice (KABP) change following TIAs were the most common types of evidence gathered by the studies in this review. Overall, the studies that measured satisfaction found that staff reported the TIC implementation activities to be beneficial and rated them positively (Bartlett \& Rushovich, 2018; Conners-Burrow et al., 2013; Hanson et al., 2019; Kramer et al., 2013; Kuhn et al., 2019; Parker et al., 2019). In studies measuring KABP change, researchers generally found statistically significant increases and improvements in KABP following TIAs (Baker et al., 2018; Barnett et al., 2018; Bartlett \& Rushovich, 2018; Brown et al., 2012; Conners-Burrow et al., 2013; Denison et al., 2018; Dierkhising \& Kerig, 2018; Dueweke et al., 2019; Hanson et al., 2019; Kenny et al., 2017; Kramer et al., 2013; Kuhn et al., 2019; McIntyre et al., 2019; Parker et al., 2019; Schiff et al., 2017; Shamblin et al., 2016; Weiss et al., 2017). However, one study (Beidas et al., 2016) found no difference in knowledge following training and several studies found that despite being significant, the increases in KABP variables were still quite modest (Conners-Burrow et al., 2013; Dueweke et al., 2019; Kramer et al., 2013).

Longitudinal studies. Studies that followed up to explore whether improvements or increases in KABP were maintained over time generated mixed results. For example, Kenny et al. (2017), Kramer et al. (2013) and Redd et al. (2017) found that they were maintained while Beidas et al. (2016) and Jankowski et al. (2018) found that they decreased at follow-up. These mixed results may be influenced by the degree to which the interventions included active and experiential learning opportunities, provided ongoing coaching and supervision and addressed organizational culture, structure and policy (Beidas \& Kendall, 2010). The dose of exposure to TIAs also seems important. Barnett et al. (2018) found a correlation between the dose of participation in training and reflective practice groups with self-reported trauma knowledge, suggesting that more engagement with TIAs may increase the level of uptake.

Staff-related variables and KABP change. Some of the studies assessing KABP change looked at how staffrelated variables may be impacting the level of uptake. However, this is an undeveloped area of research and the findings that were generated were mixed. Research into the ways that demographic variables like gender and age affect TIC uptake in this scholarship seems to remain undeveloped and inconclusive, as does research into the impact of vocational variables like level and type of education, years of experience, or role in the organization. There were also mixed findings when it came to understanding how staff members who scored the lowest on pre-tests fared post-TIAs. For example, Denison et al. (2018) found that those who changed their attitudes the most were those who had the least favorable attitudes towards TIC pre-TIAs while Kramer et al. (2013) found that supervisors who gained the most knowledge from training were more likely to change their behavior. However, Mclntyre et al. (2019) found that among teachers who reported low "system fit" between TIC and their schools, gains in knowledge were associated with decreased acceptability of TIC. They stated that "increased knowledge may have highlighted system barriers rather than supports and ultimately led to lower acceptability scores, contradicting the intended effect of the training" (p. 100).

Studying the ways that psychological variables, like how staff member's trauma histories impact uptake of TIC represents an emerging area of TIC implementation inquiry. While none of the articles in this review set out to better understand how this factor affects TIC uptake, several articles discussed anecdotally how staff's trauma histories impacted TIAs, and a few found that this theme emerged in their qualitative data. For example, Barnett et al. (2018) stated:

Although high rates of personal trauma among residential and other human service staff is well documented in the literature, we were still alarmed by the number of staff who revealed their own traumatic histories and traumatic stress symptoms, either during the trainings, supervision groups, or written assignments [...] No staff were debilitated by these memories and insights; rather, they felt empowered and more effective in their job when allowed to process these experiences in a safe 
environment. Still, devoting more resources, such as outreach and assistance from an Employee Assistance Program, to help staff manage their own traumatic and secondary traumatic stress may have further strengthened the program (p. 110).

Ignoring these psychological factors at play in TIC uptake as well as staff's needs for more support during TIAs represents a major gap in TIC implementation research and practice. Further, research and implementation practice should be more sensitive to staff's needs and more intentional about including a focus on how factors like these impact TIC uptake as well as other facets of TIC implementation.

\section{Staff outcomes}

While a trauma lens was largely missing in the research that looked at KABP change, there were two studies that looked at levels of secondary traumatic stress among staff post-TIAs (Baker et al., 2018; Damian et al., 2017). Both of these studies found an increase in secondary trauma levels among staff post-TIAs but interpreted this as an increase in awareness rather than an increase in actual distress. In the qualitative data collected by Baker et al. (2018), they found that participants reported that the training not only helped them identify and understand secondary trauma but validated the experience of secondary trauma as systemic rather than the result of individual deficiencies. Jacobowitz et al. (2015) found that post-traumatic stress symptoms among staff increased as the length of time between attending TIC meetings increased, suggesting that either attendance at meetings is a protective factor or that staff struggling with these symptoms tended to attend meetings less frequently.

With respect to other types of staff-related outcomes of TIAs, there were again mixed results as well as heterogenous variables being studied. For example, Barnett et al. (2018) found no change in staff retention rates, felt sense of safety at work or job satisfaction following a fairly robust TIC implementation initiative. However, Forrest et al. (2018) found "maintained decreases in both staff restraint-related injuries and number of, and average payout of worker's compensation claims, which are likely correlated" (p. 278). Given the wide range of the types of staff outcomes being studied, which range from secondary stress, turnover, job satisfaction to staff injuries, it may take time for enough evidence to be generated about each of them for a clearer picture to emerge about how each is impacted by different types of TIAs in different contexts.

\section{Child and youth outcomes}

Some of the TIC research studied the ways that the children and youth being served were impacted following TIAs. Again, they reported on a highly diverse set of variables but generated promising results, which hinted at the ways that TIC could bolster child and youth resilience. The Ashby et al. (2019) study of TIC implementation at an obstetric and pediatric medical home for pregnant and parenting adolescent girls and their children found significant improvements in attendance at prenatal appointments and in the birthweight of babies born to girls in the program. Forrest et al. (2018) found decreases in restraint-related injuries in their clients and Murphy et al. (2017) found greater placement stability. Several studies observed improvements in many aspects of children and youth's overall health, functioning and well-being (Hodgdon et al., 2013; Murphy et al., 2017; Pollastri et al., 2016; Rivard et al., 2005; Shamblin et al., 2016). These studies looked at variables such as emotional regulation, externalizing and internalizing behaviors, PTSD symptoms, somatic complaints, rates of self-harm, and school functioning with limited overlap between the outcomes being measured between studies, even between those from the same sectors. Sadly, none of the studies in this review sought direct feedback about the changes being made from the children and youth that the departments, organizations or systems were serving. The studies generally chose a more indirect route by exploring child and youth outcomes through administrative data or chart review. Consulting directly with the children and youth being served about the changes being made may be an important direction for future research.

\section{Organizational / Systemic outcomes}

Overall, there was again little consistency in terms of the variables being measured and results being generated, except when it came to scholarship about the impact of TIAs on seclusion and restraint practices in inpatient and residential facilities. TIC implementation initiatives that targeted reductions in seclusion and chemical/physical restraint practices seemed quite successful. All studies that collected data on these practices found a reduction postimplementation that was generally maintained at follow-up (Azeem et al., 2011; Forrest et al., 2018; Goetz \& TaylorTrujillo, 2012; Hambrick et al., 2018; Hodgdon et al., 2013; Pollastri et al., 2016; Regan, 2010). Moreover, some of these reductions were linked to organizational culture change following TIAs (Drabble et al., 2013; Goetz \& Taylor-Trujillo, 2012; Regan, 2010; Rivard et al., 2004, 2005). For example, Goetz and Taylor-Trujillo (2012) found that: 
the culture of the units moved from one of quickly subduing a patient who is aggressive to allowing whatever time was necessary to work with a patient to reduce his or her anxiety and fear, or anger. The staff members were taught to use a "show of support" instead of a "show of force" with patients (p. 99).

These changes were also linked to significant cost savings. Hambrick et al. (2018) state that over one million dollars may have been saved over six-years while Pollastri et al. (2016) estimate $\$ 339,703$ in savings over four years. Both of these studies used estimation guides developed by Lebel and Goldstein in 2005 that suggested that the average restraint in an inpatient facility costs approximately $\$ 350$ due to requiring approximately 12 person hours. Studies that looked at critical and violent incidents also reported overall decreases following TIAs (Baetz et al., 2019; Barnett et al., 2018; Goetz \&Taylor-Trujillo, 2012).

Of the studies that found mostly improvements in organizational and systematic TIC outcomes, Beidas et al. (2016) found an increase in the number of providers of TSIs in the area and an increase in youth identified and treated. Moreover, Redd et al. (2017) reported that fidelity measures of the TIC model used increased over time and Shamblin et al. (2016) found a decrease in negative attributes of the preschool learning environment. However, many studies reported having more difficulty creating a meaningful impact on the organizational or systemic contexts (Damian et al., 2019; Hummer et al., 2010; Jankowski et al., 2018; Lang et al., 2016; Williams et al., 2017). For example, Hummer et al. (2010) found that while many of the TIC principles were beginning to be operationalized among the agencies being studied, most agencies had not yet acted on creating supports or resources for staff experiencing secondary trauma as a result of their work with complex trauma-impacted children and youth. Qualitative evidence from the Watt (2017) study revealed how the TIC approach can sometimes clash with the conventional approach to youth mental health in the US, which is based on the Diagnostic and Statistical Manual of Mental Disorders (DSM). Watt reports that their findings reveal that "when the two frameworks clash, the DSM system ultimately ignores, defeats, or co-opts the trauma perspective in order to maintain dominance" (p. 401). This study highlights the structural barriers to TIC implementation and the challenges associated with fostering a paradigm shift in service delivery philosophy.

Reporting on organizational and systemic outcomes of TIC implementation helped to triangulate the selfreports of KABP change with data that reflected observable changes to behaviour and practice, such as administrative data. When present, this triangulation provided insight into how much the self-reported changes to KABP translated into observable change to behaviour, practice and organizational climate. Outside of the seclusion- and restraint-based scholarship, it revealed that observable changes to KABP were more limited than those that staff self-reported. For example, Dueweke et al. (2019) found that despite a statistically significant increase in KABP among pediatric residents following TIAs, there was minimal change to resident's screening and referral practices as measured by administrative data. Future research should consider including at least one type of organizational or systemic indicator that could triangulate self-reports of KABP change.

\section{Characteristics of the TIC implementation process}

Complexity of the process. Most articles, especially those studying MCls and CCls, described TIC implementation and the process of studying TIC implementation as resource intensive, iterative and highly complex. For example, an article from the youth justice sector states: "the move towards trauma-informed care (TIC) is neither simple nor straightforward" (Collin-Vézina et al., 2019, p. 634). Moving forward, it may be helpful to conceptualize TIC implementation through the lens of complexity science which understands organizational change as an unpredictable and messy process (Day, 2020). As Bryson et al. (2017) state in their systemic review of TIC in youth inpatient and residential settings, "we are dealing with complex social interventions which act on complex social systems. These are not magic bullets which will always hit their target, but programmes whose effects are crucially dependent on context and implementation" (p. 9). In light of this, a comprehensive, whole-system approach to the implementation process that is theoretically grounded, developmentally informed and is flexible enough to be adapted to each organization's unique context was recommended throughout this literature.

Facilitators of the process. The most salient facilitators of TIC implementation in the CYSSs that emerged from this review are: 1) commitment from senior leadership, 2) ongoing staff support, and 3) intra-organizational and inter-organizational collaboration. Implementation initiatives that only engaged front-line staff generally had a limited impact as buy-in from senior leadership was required to make meaningful changes to organizational culture, structure and policy (Damian et al., 2019). When all levels of the organization or system were engaged and on board, meaningful changes were more easily achieved (Barnett et al., 2018; Lang et al., 2016; Williams et al., 2017). Moreover, the Middleton 
et al. (2015) qualitative study of leadership styles found that successful leaders of Sanctuary Model implementation used a transformational leadership style which promoted open dialogue, the empowerment of staff and the interrogation of commonly held assumptions. One-off training interventions generated limited impacts, as staff members generally needed ongoing coaching, mentoring, supervision and/or training to support the process of integrating new knowledge and applying it in challenging practice contexts (Ashby et al., 2019; Azeem et al., 2011; Bartlett \& Rushovich, 2018; Collin-Vézina et al., 2019; Hambrick et al., 2018; Lang et al., 2016; Price et al., 2019). The development of a shared TIC language and perspective was often discussed as an outcome of TIAs and also as an important step towards improved communication and collaboration within and between service providing organizations (Bartlett \& Rushovich, 2018; Barto et al., 2018; Beidas et al., 2016; Jankowski et al., 2018; Redd et al., 2017). Effective communication and collaboration was necessary for facing the complexity of the challenges associated with implementing TIC throughout a service system and with tailoring the TIC frameworks to local needs.

Barriers to the process. The most common barriers to TIC implementation included scarce resources, time constraints and competing demands (Damian et al., 2018; Dueweke et al., 2019; Fraser et al., 2015; Jankowski et al., 2018; Kramer et al., 2013). Taking time away from heavy caseloads to engage in TIAs was difficult to navigate when organizational funding often depended on the volume of clients being served. Some of the studies also illuminated barriers that were structural and emerged from inequitable power relations. Regan (2010) reported on the power struggles that emerged between the nurses and physicians on an inpatient unit when nurses started resisting the use of chemical restraints following TIAs. The physicians on the unit were not accustomed to their orders for intramuscular injection not being met with unquestioning obedience. McCorkle et al. (2005) discussed the racial power dynamics between TIC consultants, trainees and the children and youth being served, reporting that a participant made the following comment during a training session: "This project sounds like several white people telling a whole lot of black people how to care for a whole lot of black children" (p. 130). Exploring the ways that TIC disrupts or reinforces existing power relations within organizations is an important direction for future research.

\section{Conclusion}

The variability in the types of TIC implementation initiatives being studied, the types of evidence being collected and in the findings being generated points to the complexity and infancy of this research area. The variability in the quality, scope, scale and transparency of TIC implementation research and practice may reflect the rapid expansion of the TIC consultation and training industry and the need for more reflexivity and thoughtfulness as the TIC field grows. Systemic change across a wide range of sectors is nothing short of complex and it is clear that for the scholarship to progress, researchers will need to be more creative in developing methodologies that are more suitable for capturing the complexity of organizational and systemic change. In order to do so, thinking outside the box of the EBP hierarchy of evidence will be important, especially as the TIC framework evolves to include a better response to structural violence. Working towards a consensus about the core components of TIC implementation should also be a priority given the limited impacts of LCls. However, it is important to balance the need for clearer guidelines with the need for these guidelines to be tailored to the unique needs of each different service provision context. Furthermore, as this field matures, it would be advisable to increase the transparency regarding the characteristics of the change initiatives being studied. In order to generate a nuanced knowledge base about how to best create change in a variety of different contexts, more details about each aspect of the change initiatives will be needed.

While certain process-related factors that contribute to successful implementation are becoming clearer, the outcome-oriented TIC scholarship was highly variable and had yet to produce conclusive evidence about many aspects of TIC implementation. However, the preliminary outcome-based evidence suggests that when comprehensive approaches to implementation are taken, harmful, coercive and violent practices and incidents are reduced, and child and youth resilience and well-being is bolstered. Other preliminary evidence points to the relatively consistent increase in self-reports of TIC-favorable knowledge, competency, attitudes and beliefs following TIAs. However, research shows that these increases are often modest, not always maintained over time and not easily translated into changes in behaviour, practice and organizational climate due to a wide variety of barriers. In order to address these barriers, the evidence suggests that TIC implementation requires a comprehensive approach that includes commitment from senior leadership, ongoing support, and collaboration within and between service providing organizations and systems. Before investing in TIC training, organizational leaders should consider the readiness of the organization to change and the capacity and resources available to support TIAs over time. The booming TIC training industry may be 
overselling the benefits of one-time training initiatives and a little training does not a trauma-informed organization make.

\section{Funding}

The author(s) disclosed receipt of the following financial support for the research, authorship, and/or publication of this article: This initiative was funded by the Fonds de recherche du Québec - Société et culture.

\section{Conflict of interest}

The author declared no potential conflicts of interest with respect to the research, authorship, and/or publication of this article.

\section{References}

References marked with an asterisk (*) indicate studies included in the scoping review.

*Akin, B. A., Strolin-Goltzman, J., \& Collins-Camargo, C. (2017). Successes and challenges in developing trauma-informed child welfare systems: A real-world case study of exploration and initial implementation. Children and Youth Services Review, 82, 42-52. https://doi.org/10.1016/j.childyouth.2017.09.007

Arksey, H., \& O'Malley, L. (2005). Scoping studies: Towards a methodological framework. International Journal of Social Research Methodology, 8(1), 19-32. https://doi.org/10.1080/1364557032000119616

*Ashby, B. D., Ehmer, A. C., \& Scott, S. M. (2019). Trauma-informed care in a patient-centered medical home for adolescent mothers and their children. Psychological Services, 16(1), 67-74. https://doi.org/10.1037/ser0000315

*Azeem, M. W., Aujla, A., Rammerth, M., Binsfeld, G., \& Jones, R. B. (2011). Effectiveness of six core strategies based on trauma informed care in reducing seclusions and restraints at a child and adolescent psychiatric hospital. Journal of Child and Adolescent Psychiatric Nursing, 24(1), 11-15. https://doi.org/10.1111/j.1744-6171.2010.00262.x

*Baetz, C. L., Surko, M., Moaveni, M., McNair, F., Bart, A., Workman, S., Tedeschi, F., Havens, J., Guo, F., Quinlan, C., \& Horwitz, S. M. (2019). Impact of a trauma-informed intervention for youth and staff on rates of violence in juvenile detention settings. Journal of Interpersonal Violence. Advance online publication. https://doi.org/10.1177/0886260519857163

Bailey, C., Klas, A., Cox, R., Bergmeier, H., Avery, J., \& Skouteris, H. (2019). Systematic review of organisation-wide, trauma-informed care models in out-of-home care settings. Health \& Social Care in the Community, 27(3), 10-22. https://doi.org/10.1111/hsc.12621

*Baker, C. N., Brown, S. M., Wilcox, P., Verlenden, J. M., Black, C. L., \& Grant, B. E. (2018). The implementation and effect of traumainformed care within residential youth services in Rural Canada: A mixed methods case study. Psychological Trauma: Theory, Research, Practice, and Policy, 106), 666-674. https://doi.org/10.1037/tra0000327

*Barnett, E. R., Yackley, C. R., \& Lich, E. S. (2018). Developing, implementing, and evaluating a trauma-informed care program within a youth residential treatment center and special needs school. Residential Treatment for Children \& Youth, 35(2), 95-113. https://doi.org/10.1080/0886571x.2018.1455559

Bartlett, J. D., Barto, B., Griffin, J. L., Fraser, J. G., Hodgdon, H., \& Bodian, R. (2016). Trauma-informed care in the Massachusetts Child Trauma Project. Child Maltreatment, 21(2), 101-112. https://doi.org/10.1177/1077559515615700

*Bartlett, J. D., \& Rushovich, B. (2018). Implementation of Trauma Systems Therapy-Foster Care in child welfare. Children and Youth Services Review, 91, 30-38. https://doi.org/10.1016/j.childyouth.2018.05.021

*Barto, B., Bartlett, J. D., Von Ende, A., Bodian, R., Norona, C. R., Griffin, J., Fraser, J. G., Kinniburgh, K., Spinazzola, J., Montagna, C., \& Todd, M. (2018). The impact of a statewide trauma-informed child welfare initiative on children's permanency and maltreatment outcomes. Child Abuse \& Neglect, 81, 149-160. https://doi.org/10.1016/j.chiabu.2018.04.023

*Beidas, R. S., Adams, D. R., Kratz, H. E., Jackson, K., Berkowitz, S., Zinny, A., Cliggitt, L.P., Dewitt, K.L., Skriner, L., \& Evans, A. (2016). Lessons learned while building a trauma-informed public behavioral health system in the City of Philadelphia. Evaluation and Program Planning, 59, 21-32. https://doi.org/10.1016/j.evalprogplan.2016.07.004

Beidas, R. S., \& Kendall, P. C. (2010). Training therapists in evidence-based practice: A critical review of studies from a systemscontextual perspective. Clinical Psychology: Science and Practice, 1711), 1-30. https://doi.org/10.1111/j.14682850.2009.01187.x

Birnbaum, S. (2019). Confronting the social determinants of health: Has the language of trauma informed care become a defense mechanism? Issues in Mental Health Nursing, 4066), 476-481. https://doi.org/10.1080/01612840.2018.1563256 
Blaustein, M., \& Kinniburgh, K. M. (2010). Treating traumatic stress in children and adolescents: How to foster resilience through attachment, self-regulation, and competency. Guilford Press.

Bloom, S. L., \& Farragher, B. (2010). Destroying sanctuary: The crisis in human service delivery systems. Oxford University Press. https://doi.org/10.1093/acprof:oso/9780195374803.001.0001

Bloom, S. L., \& Farragher, B. (2013). Restoring sanctuary: A new operating system for trauma-informed systems of care: Oxford University Press. https://doi.org/10.1093/acprof:oso/9780199796366.001.0001

*Brown, S. M., Baker, C. N., \& Wilcox, P. (2012). Risking connection trauma training: A pathway toward trauma-informed care in child congregate care settings. Psychological Trauma: Theory, Research, Practice, and Policy, 4(5), $507-515$. https://doi.org/10.1037/a0025269

Bryson, S. A., Gauvin, E., Jamieson, A., Rathgeber, M., Faulkner-Gibson, L., Bell, S., Davidson, J., Russel, J., \& Burke, S. (2017). What are effective strategies for implementing trauma-informed care in youth inpatient psychiatric and residential treatment settings? A realist systematic review. International Journal of Mental Health Systems, 11(1), Article 36. https://doi.org/10.1186/s13033017-0137-3

Cloitre, M., Stolbach, B. C., Herman, J. L., Kolk, B. V. D., Pynoos, R., Wang, J., \& Petkova, E. (2009). A developmental approach to complex PTSD: Childhood and adult cumulative trauma as predictors of symptom complexity. Journal of Traumatic Stress, 22(5), 399408. https://doi.org/10.1002/jts.20444

*Collin-Vézina, D., McNamee, S., Brazeau, C., \& Laurier, C. (2019). Initial implementation of the ARC framework in juvenile justice settings. Journal of Aggression Maltreatment \& Trauma, 28(5), 631-654. https://doi.org/10.1080/10926771.2019.1583709

*Conners-Burrow, N. A., Kramer, T. L., Sigel, B. A., Helpenstill, K., Sievers, C., \& McKelvey, L. (2013). Trauma-informed care training in a child welfare system: Moving it to the front line. Children \& Youth Services Review, 35(11), $1830-1835$. https://doi.org/10.1016/j.childyouth.2013.08.013

${ }^{*}$ Counts, J. M., Gillam, R. J., Perico, S., \& Eggers, K. L. (2017). Lemonade for life: A pilot study on a hope-infused, trauma-informed approach to help families understand their past and focus on the future. Children and Youth Services Review, 79, $228-234$. https://doi.org/10.1016/j.childyouth.2017.05.036

Courtois, C. A. (2008). Complex trauma, complex reactions: Assessment and treatment. Psychological Trauma: Theory, Research, Practice, and Policy, S(1), 86-100. https://doi.org/10.1037/1942-9681.S.1.86

${ }^{*}$ Crandal, B. R., Hazen, A. L., \& Reutz, J. R. (2017). Identifying trauma-related and mental health needs: The implementation of screening in California's child welfare systems. Advances in Social Work, 18(1), 335-348. https://doi.org/10.18060/21278

*Damian, A. J., Gallo, J., Leaf, P., \& Mendelson, T. (2017). Organizational and provider level factors in implementation of traumainformed care after a city-wide training: an explanatory mixed methods assessment. Health Services Research, 171), 750. https://doi.org/10.1186/s12913-017-2695-0

*Damian, A. J., Gallo, J. J., \& Mendelson, T. (2018). Barriers and facilitators for access to mental health services by traumatized youth. Children and Youth Services Review, 85, 273-278. https://doi.org/10.1016/j.childyouth.2018.01.003

*Damian, A. J., Mendelson, T., Bowie, J., \& Gallo, J. J. (2019). A mixed methods exploratory assessment of the usefulness of Baltimore City Health Department's trauma-informed care training intervention. American Journal of Orthopsychiatry, 89(2), 228-236. https://doi.org/10.1037/ort0000357

Day, A. (2020). Disruption, change and transformation in organisations: A human relations perspective. Routledge.

*Denison, M., Gerney, A., Barbuti Van Leuken, J., \& Conklin, J. (2018). The attitudes and knowledge of residential treatment center staff members working with adolescents who have experienced trauma. Residential Treatment for Children \& Youth, 35(2), 114138. https://doi.org/10.1080/0886571x.2018.1458689

*Dierkhising, C. B., \& Kerig, P. K. (2018). Pilot evaluation of a university-based training in trauma-informed services for gang intervention workers. Journal of Aggression, Maltreatment and Trauma, 27(3), $291-308$. https://doi.org/10.1080/10926771.2017.1382634

*Drabble, L. A., Jones, S., \& Brown, V. (2013). Advancing trauma-informed systems change in a family drug treatment court context. Journal of Social Work Practice in the Addictions, 13(1), 91-113. https://doi.org/10.1080/1533256x.2012.756341

*Dueweke, A. R., Hanson, R. F., Wallis, E., Fanguy, E., \& Newman, C. (2019). Training pediatric primary care residents in trauma-informed care: A feasibility trial. Clinical Pediatrics, 58(11-12), 1239-1249. https://doi.org/10.1177/0009922819859868

Elliott, D. E., Bjelajac, P., Fallot, R. D., Markoff, L. S., \& Reed, B. G. (2005). Trauma-informed or trauma-denied: Principles and implementation of trauma-informed services for women. Journal of Community Psychology, 33(4), $461-477$. https://doi.org/10.1002/jcop.20063

*Esaki, N., Hopson, L. M., \& Middleton, J. S. (2018). Sanctuary Model implementation from the perspective of indirect care staff. Families in Society: The Journal of Contemporary Social Services, 95(4), 261-268. https://doi.org/10.1606/1044-3894.2014.95.31 
*Forrest, S., Gervais, R., Lord, K. A., Sposato, A., Martin, L., Beserra, K., \& Spinazzola, J. (2018). Building communities of care: A comprehensive model for trauma-informed youth capacity building and behavior management in residential services. Residential Treatment for Children \& Youth, 35(4), 265-285. https://doi.org/10.1080/0886571x.2018.1497930

Ford, J. D., \& Blaustein, M. E. (2013). Systemic self-regulation: A framework for trauma-informed services in residential juvenile justice programs. Journal of Family Violence, 28(7), 665-677. https://doi.org/10.1007/s10896-013-9538-5

*Fraser, J. G., Griffin, J. L., Bartlett, J. D., Barto, B. L., Lo, C., Wenz-Gross, M., Spinazzola, J., Bodian, R.A., \& Nisenbaum, J. M. (2015). Implementation of a workforce initiative to build trauma-informed child welfare practice and services: Findings from the Massachusetts Child Trauma Project. Children and Youth Services Review, 44, $233-242$. https://doi.org/10.1016/j.childyouth.2014.06.016

Garrard, J. (2017). Health sciences literature review made easy: The matrix method. Jones \& Bartlett Learning.

*Goetz, S. B., \& Taylor-Trujillo, A. (2012). A change in culture: Violence prevention in an acute behavioral health setting. Journal of the American Psychiatric Nurses Association, 18(2), 96-103. https://doi.org/10.1177/1078390312439469

*Hambrick, E. P., Brawner, T. W., Perry, B. D., Wang, E., Griffin, G., Demarco, T., Grove, T., Maikoetter, M., O'Malley, D., Paxton, D., Freedle, L., Freidman, J., Mackenzie, J., Perry, K.M., Cudney, P., Hartman, J., Kuh, E., Morris, J., \& Polales, C. (2018). Restraint and critical incident reduction following introduction of the Neurosequential Model of Therapeutics (NMT). Residential Treatment for Children \& Youth, 35(1), 2-23. https://doi.org/10.1080/0886571x.2018.1425651

Hanson, R. F., \& Lang, J. (2016). A critical look at trauma-informed care among agencies and systems serving maltreated youth and their families. Child Maltreatment, 21(2), 95-100. https://doi.org/10.1177/1077559516635274

*Hanson, R. F., Saunders, B. E., Ralston, E., Moreland, A. D., Peer, S. O., \& Fitzgerald, M. M. (2019). Statewide implementation of child trauma-focused practices using the community-based learning collaborative model. Psychological Services, 16(1), $170-181$. https://doi.org/10.1037/ser0000319

Harris, M., \& Fallot, R. D. (2001). Envisioning a trauma-informed service system: a vital paradigm shift. New Directions for Mental Health Services, 2001(89), 3-22. https://doi.org/10.1002/yd.23320018903

*Hodgdon, H. B., Kinniburgh, K., Gabowitz, D., Blaustein, M. E., \& Spinazzola, J. (2013). Development and Implementation of TraumaInformed Programming in Youth Residential Treatment Centers Using the ARC Framework. Journal of Family Violence, 28(7), 679-692. https://doi.org/10.1007/s10896-013-9531-z

Hopper, E. K., Bassuk, E. L., \& Olivet, J. (2010). Shelter from the storm: Trauma-informed care in homelessness services settings. Open Health Services and Policy Journal, 3(2), 80-100.

*Hoysted, C., Jobson, L., \& Alisic, E. (2019). A pilot randomized controlled trial evaluating a web-based training program on pediatric medical traumatic stress and trauma-informed care for emergency department staff. Psychological Services, 16(1), 38-47. https://doi.org/10.1037/ser0000247

*Hummer, V. L., Dollard, N., Robst, J., \& Armstrong, M. I. (2010). Innovations in implementation of trauma-informed care practices in youth residential treatment: A curriculum for organizational change. Child Welfare, 89(2), 79-95.

*Jacobowitz, W., Moran, C., Best, C., \& Mensah, L. (2015). Post-traumatic stress, trauma-informed care, and compassion fatigue in psychiatric hospital staff: A correlational study. Issues in Mental Health Nursing, 36(11), $890-899$. https://doi.org/10.3109/01612840.2015.1055020

*Jankowski, M. K., Schifferdecker, K. E., Butcher, R. L., Foster-Johnson, L., \& Barnett, E. R. (2018). Effectiveness of a trauma-informed care initiative in a state child welfare system: A randomized study. Child Maltreatment, 24(1), 86-97. https://doi.org/10.1177/1077559518796336

*Kenny, M. C., Vazquez, A., Long, H., \& Thompson, D. (2017). Implementation and program evaluation of trauma-informed care training across state child advocacy centers: An exploratory study. Children and Youth Services Review, 73, 15-23. https://doi.org/10.1016/j.childyouth.2016.11.030

*Kramer, T. L., Sigel, B. A., Conners-Burrow, N. A., Savary, P. E., \& Tempel, A. (2013). A statewide introduction of trauma-informed care in a child welfare system. Children and Youth Services Review, 35(1), 19-24. https://doi.org/10.1016/j.childyouth.2012.10.014

*Kuhn, T. M., Cyperski, M. A., Shaffer, A. M., Gracey, K. A., Adams, M. K., Billings, G. M., \& Ebert, J. S. (2019). Installing trauma-informed care through the Tennessee Child Protective Services Academy. Psychological Services, 16(1), $143-152$. https://doi.org/10.1037/ser0000320

*Lang, J. M., Campbell, K., Shanley, P., Crusto, C. A., \& Connell, C. M. (2016). Building capacity for trauma-informed care in the child welfare system. Child Maltreatment, 21(2), 113-124. https://doi.org/10.1177/1077559516635273

Levac, D., Colquhoun, H., \& O'Brien, K. K. (2010). Scoping studies: advancing the methodology. Implementation Science, 5(1), Article 69. https://doi.org/10.1186/1748-5908-5-69 
Levine, S., Varcoe, C., \& Browne, A. J. (2020). "We went as a team closer to the truth": impacts of interprofessional education on traumaand violence- informed care for staff in primary care settings. Journal of Interprofessional Care. Advance online publication. https://doi.org/10.1080/13561820.2019.1708871

Markiewicz, J., Ebert, L., Ling, D., Amaya-Jackson, L., \& Kisiel, C. (2006). Learning Collaborative Toolkit. National Center for Child Traumatic Stress. http://www.ncdsv.org/images/NCTSN_LearningCollaborativeToolkit.pdf

*McCorkle, D., Peacock, C., \& Campus, W. (2005). Trauma and the Isms - A herd of elephants in the room: A training vignette. Therapeutic Community: International Journal for Therapeutic and Supportive Organizations, 26(1), 127-133.

*McIntyre, E. M., Baker, C. N., \& Overstreet, S. (2019). Evaluating foundational professional development training for trauma-informed approaches in schools. Psychological Services, 16(1), 95-102. https://doi.org/10.1037/ser0000312

*Middleton, J., Harvey, S., \& Esaki, N. (2015). Transformational leadership and organizational change: How do leaders approach trauma-informed organizational change...Twice? Families in Society, 96(3), 155-163. https://doi.org/10.1606/10443894.2015.96.21

Munn, Z., Peters, M. D. J., Stern, C., Tufanaru, C., McArthur, A., \& Aromataris, E. (2018). Systematic review or scoping review? Guidance for authors when choosing between a systematic or scoping review approach. BMC Medical Research Methodology, 18(1). https://doi.org/10.1186/s12874-018-0611-x

*Murphy, K., Moore, K. A., Redd, Z., \& Malm, K. (2017). Trauma-informed child welfare systems and children's well-being: A longitudinal evaluation of KVC's bridging the way home initiative. Children and Youth Services Review, 75, 23-34. https://doi.org/10.1016/j.childyouth.2017.02.008

National Child Traumatic Stress Network. (2013). Child Welfare Trauma Training Toolkit. https://www.nctsn.org/resources/childwelfare-trauma-training-toolkit

Oudshoorn, J. (2015). Trauma-informed youth justice in Canada: A new framework toward a kinder future. Canadian Scholars' Press.

*Palfrey, N., Reay, R. E., Aplin, V., Cubis, J. C., McAndrew, V., Riordan, D. M., \& Raphael, B. (2019). Achieving service change through the implementation of a trauma-informed care training program within a mental health service. Community Mental Health Journal, 55(3), 467-475. https://doi.org/10.1007/s10597-018-0272-6

*Parker, J., Olson, S., \& Bunde, J. (2019). The impact of trauma-based training on educators. Journal of Child and Adolescent Trauma. Advance online publication. https://doi.org/10.1007/s40653-019-00261-5

Perry, B. D. (2009). Examining child maltreatment through a neurodevelopmental lens: Clinical applications of the neurosequential model of therapeutics. Journal of Loss and Trauma, 14(4), 240-255. https://doi.org/10.1080/15325020903004350

Peters, M. D. J., Godfrey, C. M., Khalil, H., Mclnerney, P., Parker, D., \& Soares, C. B. (2015). Guidance for conducting systematic scoping reviews. International Journal of Evidence-Based Healthcare, 13(3), 141-146. https://doi.org/10.1097/xeb.0000000000000050

*Pollastri, A. R., Lieberman, R. E., Boldt, S. L., \& Ablon, J. S. (2016). Minimizing seclusion and restraint in youth residential and day treatment through site-wide implementation of collaborative problem solving. Residential Treatment for Children \& Youth, 33(3-4), 186-205. https://doi.org/10.1080/0886571x.2016.1188340

*Price, J., Genuario, K., Romeo, D., Pruden, K., Elwell, S., Matwiejewicz, D., Freidlander, E., \& Jaszczyszyn, D. (2019). Implementation of a standardized screening program for risk of posttraumatic stress disorder among youth hospitalized with injury. Psychological Services, 16(1), 48-57. https://doi.org/10.1037/ser0000271

Purtle, J. (2020). Systematic review of evaluations of trauma-informed organizational interventions that include staff trainings. Trauma, Violence, \& Abuse, 21(4), 725-740. https://doi.org/10.1177/1524838018791304

*Redd, Z., Malm, K., Moore, K., Murphy, K., \& Beltz, M. (2017). KVC's Bridging the Way Home: An innovative approach to the application of trauma systems therapy in child welfare. Children and Youth Services Review, 76, 170-180. https://doi.org/10.1016/j.childyouth.2017.02.013

*Regan, K. (2010). Trauma informed care on an inpatient pediatric psychiatric unit and the emergence of ethical dilemmas as nurses evolved their practice. Issues in Mental Health Nursing, 37(3), 216-222. https://doi.org/10.3109/01612840903315841

*Rivard, J. C., McCorkle, D., Duncan, M. E., Pasquale, L. E., Bloom, S. L., \& Abramovitz, R. (2004). Implementing a trauma recovery framework for youths in residential treatment. Child and Adolescent Social Work Journal, 21(5), 529-550. https://doi.org/10.1023/b:casw.0000043363.14978.e6

*Rivard, J. C., Bloom, S. L., McCorkle, D., \& Abramovitz, R. (2005). Preliminary results of a study examining the implementation and effects of a trauma recovery framework for youths in residential treatment. Therapeutic Community: The International Journal for Therapeutic and Supportive Organizations, 26(1), 83-96.

*Schiff, D. M., Zuckerman, B., Hutton, E., Genatossio, C., Michelson, C., \& Bair-Merritt, M. (2017). Development and pilot implementation of a trauma-informed care curriculum for pediatric residents. Academic Pediatrics, 177), $794-796$. https://doi.org/10.1016/j.acap.2017.03.011 
*Shamblin, S., Graham, D., \& Bianco, J. A. (2016). creating trauma-informed schools for rural Appalachia: The Partnerships Program for enhancing resiliency, confidence and workforce development in early childhood education. School Mental Health, 8(1), 189200. https://doi.org/10.1007/s12310-016-9181-4

*Spehr, M. K., Zeno, R., Warren, B., Lusk, P., \& Masciola, R. (2019). Social-emotional screening protocol implementation: A traumainformed response for young children in child welfare. Journal of Pediatric Health Care, 33(6), 675-683. https://doi.org/10.1016/j.pedhc.2019.05.003

Substance Abuse and Mental Health Services Administration. (2014). SAMHSA's concept of trauma and guidance for a traumainformed approach (HHS Publication No. 14-4884). https://ncsacw.samhsa.gov/userfiles/files/SAMHSA_Trauma.pdf

Thomas, M. S., Crosby, S., \& Vanderhaar, J. (2019). Trauma-informed practices in schools across two decades: An interdisciplinary review of research. Review of Research in Education, 43(1), 422-452. https://doi.org/10.3102/0091732X18821123

van der Kolk, B. A., Roth, S., Pelcovitz, D., Sunday, S., \& Spinazzola, J. (2005). Disorders of extreme stress: The empirical foundation of a complex adaptation to trauma. Journal of Traumatic Stress, 18(5), 389-399. https://doi.org/10.1002/jts.20047

*Watt, T. (2017). Paradigm shifts don't come easy: Confrontations between the trauma perspective and the DSM in mental health treatment for abused and neglected children. Journal of Child and Adolescent Trauma, 10(4), $395-403$. https://doi.org/10.1007/s40653-017-0178-4

*Weiss, D., Kassam-Adams, N., Murray, C., Kohser, K. L., Fein, J. A., Winston, F. K., \& Marsac, M. L. (2017). Application of a framework to implement trauma-informed care throughout a pediatric health care network. Journal of Continuing Education in the Health Professions, 371), 55-60. https://doi.org/10.1097/CEH.0000000000000140

*Williams, T. M., \& Smith, G. P. (2017). Does training change practice? A survey of clinicians and managers one year after training in trauma-informed care. Journal of Mental Health Training, Education \& Practice, 12(3), 188-198. https://doi.org/10.1108/JMHTEP-02-2016-0016 